\title{
Next-generation sequencing of southern African Crimean- Congo haemorrhagic fever virus isolates reveals a high frequency of $M$ segment reassortment
}

\author{
D. GOEDHALS ${ }^{1}$, P. A. BESTER ${ }^{1}$, J. T. PAWESKA ${ }^{2,3}$, R. SWANEPOEL ${ }^{4}$ \\ AND F. J. BURT ${ }^{1 *}$ \\ ${ }^{1}$ Department of Medical Microbiology and Virology, National Health Laboratory Service/University of the Free \\ State, Bloemfontein, South Africa \\ ${ }^{2}$ Centre for Emerging and Zoonotic Pathogens, National Institute for Communicable Diseases, National Health \\ Laboratory Service, Johannesburg, South Africa \\ ${ }^{3}$ School of Pathology, Faculty of Health Sciences, University of the Witswatersrand, South Africa \\ ${ }^{4}$ Zoonoses Research Unit, Department of Medical Virology, University of Pretoria, South Africa
}

Received 11 December 2013; Final revision 7 March 2014; Accepted 14 March 2014; first published online 1 May 2014

\section{SUMMARY}

Crimean Congo haemorrhagic fever virus (CCHFV) is a bunyavirus with a single-stranded RNA genome consisting of three segments (S, M, L), coding for the nucleocapsid protein, envelope glycoproteins and RNA polymerase, respectively. To date only five complete genome sequences are available from southern African isolates. Complete genome sequences were generated for 10 southern African CCHFV isolates using next-generation sequencing techniques. The maximum-likelihood method was used to generate tree topologies for 15 southern African plus 26 geographically distinct complete sequences from GenBank. M segment reassortment was identified in 10/15 southern African isolates by incongruencies in grouping compared to the $\mathrm{S}$ and $\mathrm{L}$ segments. These reassortant $\mathrm{M}$ segments cluster with isolates from Asia/Middle East, while the S and L segments cluster with strains from South/West Africa. The CCHFV M segment shows a high level of genetic diversity, while the $\mathrm{S}$ and $\mathrm{L}$ segments appear to co-evolve. The reason for the high frequency of $\mathrm{M}$ segment reassortment is not known. It has previously been suggested that $\mathrm{M}$ segment reassortment results in a virus with high fitness but a clear role in increased pathogenicity has yet to be shown.

Key words: Arboviruses, bunyaviruses, haemorrhagic fever, molecular epidemiology.

\section{INTRODUCTION}

Crimean Congo haemorrhagic fever virus (CCHFV) is a member of the family Bunyaviridae, genus Nairovirus [1]. This tick-borne zoonotic virus is associated with clinical disease ranging from a non-specific febrile illness to severe disease manifesting as haemorrhagic

\footnotetext{
* Author for correspondence: Professor F. J. Burt, Department of Medical Microbiology and Virology, National Health Laboratory Service, Faculty of Health Sciences, University of the Free State, Bloemfontein, 9301, South Africa. (Email: burtfj@ufs.ac.za)
}

fever. CCHFV has a negative-sense, single-stranded RNA genome consisting of three segments designated large (L), medium (M) and small (S). The highly conserved complementary terminal nucleotide sequences result in loosely circular RNAs which together with the nucleocapsid protein, make up the three helical nucleocapsids. The L segment encodes the viral RNA polymerase while the $\mathrm{S}$ segment encodes the viral nucleocapsid protein. The $\mathrm{M}$ segment encodes a polyprotein which undergoes proteolytic processing to yield the viral glycoproteins, Gc and Gn [2].

CCHFV has been documented in more than 30 countries of Africa, Asia, Europe and the Middle 
East, with a distribution following that of Hyalomma ticks, the principal vector of the virus [3, 4]. Since the first description of clinical disease due to CCHFV infection in South Africa in 1981, 192 cases have been confirmed [5]. However, complete S, M and L sequences are available for only four isolates collected from humans and one tick isolate from South Africa. These, as well as other published CCHFV sequences, were determined by Sanger sequencing using primer walking [6-18]. In recent years, a number of nextgeneration sequencing (NGS) methods have been developed which yield large amounts of sequencing data at relatively low cost. Although the specifics differ, these methods all make use of three steps, namely, library preparation, DNA capture and enrichment, and sequencing or detection. In the field of virology, these techniques have been employed for various purposes including the discovery of novel viruses, whole viral genome sequencing, and 'deep' sequencing to determine viral quasi-species or genome variability [19]. This study aimed to make use of NGS techniques to obtain whole genome sequences of southern African $\mathrm{CCHFV}$ isolates in order to perform genetic analysis including identification of reassortment events.

\section{METHODS}

\section{Viral isolates}

Ten CCHFV isolates obtained from patients in southern Africa between 1985 and 2008 were sequenced retrospectively. RNA was extracted and supplied by Professor J. T. Paweska, Centre for Emerging and Zoonotic Pathogens, National Institute for Communicable Diseases (NICD), Johannesburg from cell culture preparations or mouse brain suspensions. Total RNA was extracted for isolate SPU44/08 using TRIzol $^{\circledR}$ reagent (Invitrogen, USA) according to the manufacturer's instructions. The remainder of the isolates were stored at $-70{ }^{\circ} \mathrm{C}$ as freeze-dried $10 \%$ suckling mouse brain suspensions at the level of mouse brain passage $2-3$. The suspensions were inoculated into Vero cell cultures and total RNA extracted from the infected cells using the acid guanidium thiocyanatephenol-chloroform method as described previously [20]. The extracted RNA was stored at $-70^{\circ} \mathrm{C}$ until use.

\section{Reverse transcriptase-polymerase chain reaction (RT-PCR) and sequencing}

The complete $\mathrm{L}$ segment was amplified using two previously described primer pairs that generated overlapping amplicons [8]. The complete $\mathrm{M}$ segment was amplified using primers designed by Deyde et al. [8]. Existing sequences in GenBank were used to design primers SF1 and SR3 (Table 1) for amplification of the $\mathrm{S}$ segment. The respective forward primers were used to perform the reverse transcription step for each amplicon using SuperScript ${ }^{\mathrm{TM}}$ III Reverse Transcriptase (Invitrogen). PCR was performed with the Expand Long Template PCR System (Roche Diagnostics GmbH, Germany) using standard cycling conditions according to the manufacturer's instructions and an annealing temperature of $48^{\circ} \mathrm{C}$. The PCR products were gel extracted and purified using the Wizard $^{\circledR}$ SV Gel and PCR Clean-Up System (Promega, USA).

The complete genomes for 3/10 isolates (SPU431/85, SPU383/87, SPU 130/89) were determined using the Ion Torrent $\mathrm{PGM}^{\mathrm{TM}}$ sequencer (Life Technologies, USA) by the Central Analytical Facility, Stellenbosch University. For the remaining seven isolates, the $\mathrm{S}$ segment data was determined previously in the laboratory using the Big Dye ${ }^{\mathrm{TM}}$ Terminator v. 3.1 Cycle Sequencing kit (Applied Biosystems, USA) according to the manufacturer's instructions and three overlapping primer sets as described in Table 2. The $\mathrm{L}$ and $M$ segments of these isolates were sequenced at the NICD, Johannesburg using the Roche 454 GS Junior ${ }^{\mathrm{TM}}$ sequencing system (Roche Diagnostics $\mathrm{GmbH}$ ). Additional sequencing of all isolates for incomplete coverage and clarification of ambiguities, including mixed bases or nucleotide substitutions and base insertions or deletions, was performed as required using the Big Dye ${ }^{\mathrm{TM}}$ Terminator v. 3.1 Cycle Sequencing kit (Applied Biosystems). Details regarding the additional primers used are available from the corresponding author upon request.

\section{Data analysis}

Raw sequencing data was converted from SFF format to FASTQ files using the sff_extract script (available as part of seq_crumbs at http://bioinf.comav.upv.es/) and trimming and filtering of reads based on length and quality scores was then performed using PRINSEQ [21]. Sequences for the L, M and S segments available in Genbank were used to compile databases and separate the reads into $\mathrm{L}, \mathrm{M}$ and $\mathrm{S}$ segment-related data using filter_by_blast (http:// bioinf.comav.upv.es/seq_crumbs/available_crumbs.html). De novo assembly of the blast-filtered and unfiltered reads was performed using MIRA [22]. The resulting 
Table 1. PCR and sequencing primers utilized for generation of amplicons used for sequencing

\begin{tabular}{llll}
\hline \hline $\begin{array}{l}\text { Primer } \\
\text { name }\end{array}$ & $\begin{array}{l}5^{\prime}-3^{\prime} \text { position relative } \\
\text { to SPU415/85 }\end{array}$ & $5^{\prime}-3^{\prime}$ sequence & Reference \\
\hline L1F & $1-22$ & TCT CAA AGA TAT CAA TCC CCC C & Deyde, 2006 [8] \\
L1R & $7773-7752$ & TTG GCA CTA TCT TTC ATT TGA C & Deyde, 2006 [8] \\
L2F & $6137-6158$ & GAA GAG CTA TAT GAC ATA AGG C & Deyde, 2006 [8] \\
L2R & $12157-12134$ & TCT CAA AGA AAT CGT TCC CCC CAC & Deyde, 2006 [8] \\
MF & $1-18$ & TCT CAA AGA AAT ACT TGC & Deyde, 2006 [8] \\
MR & $5354-5337$ & TCT CAA AGA TAT AGT GGC & Deyde, 2006 [8] \\
SF1 & $1-21$ & TCT CAA AGA AAC ACG TGC CGC & In house \\
SR $*$ & $590-569$ & GGT TCC TTC TCC TAA TCA TGT C & In house \\
SF2 & $467-487$ & GGT TTC CGT GTC AAT GCA AAC & In house \\
SR2 & $1114-1095$ & CAT TGG GGT GCT CAG CAG AG & In house \\
SF3* & $1029-1048$ & CGA CGG TGT CAC AGT TCC TC & In house \\
SR3 & $1673-1651$ & TCT CAA AGA TAT CGT TGC CGC AC & In house \\
\hline \hline
\end{tabular}

* Primers designed for sequence determination.

Table 2. Summary of data concerning the CCHFV sequences retrieved from the GenBank database and used in the study

\begin{tabular}{|c|c|c|c|c|c|}
\hline \multirow[b]{2}{*}{ Strain } & \multicolumn{3}{|c|}{ Accession number } & \multirow{2}{*}{$\begin{array}{l}\text { Year and location } \\
\text { of isolation }\end{array}$} & \multirow{2}{*}{$\begin{array}{l}\text { Source of } \\
\text { isolate }\end{array}$} \\
\hline & $\mathrm{L}$ & $\mathrm{M}$ & $\mathrm{S}$ & & \\
\hline C68031 & DQ211616 & DQ211629 & DQ211642 & 1968 China & Sheep \\
\hline Oman & DQ211619 & DQ211632 & DQ211645 & 1997 Oman & Human \\
\hline ArD15786 & DQ211614 & DQ211627 & DQ211640 & 1972 Senegal & Goat \\
\hline ArD8194 & DQ211613 & DQ211626 & DQ211639 & 1969 Senegal & Tick \\
\hline ArD39554 & DQ211615 & DQ211628 & DQ211641 & 1984 Mauritania & Tick \\
\hline SPU415/85 & DQ211622 & DQ211635 & DQ211648 & 1985 South Africa & Human \\
\hline SPU97/85 & DQ211620 & DQ211633 & DQ211646 & 1985 South Africa & Human \\
\hline SPU103/87 & DQ211621 & DQ211634 & DQ211647 & 1987 South Africa & Human \\
\hline Drosdov & DQ211617 & DQ211630 & DQ211643 & 1967 Russia & Human \\
\hline Kashmanov & DQ211618 & DQ211631 & DQ211644 & 1967 Russia & Human \\
\hline Baghdad-12 & AY947890 & AJ538197 & AJ538196 & 1979 Iraq & Human \\
\hline Matin & AY422208 & AF467769 & AF527810 & 1976 Pakistan & Human \\
\hline Kosova Hoti & EU044832 & EU037902 & DQ133507 & 2001 Kosovo & Human \\
\hline Turkey 200310849 & DQ211623 & DQ211636 & DQ211649 & 2003 Turkey & Human \\
\hline UG3010 & DQ211624 & DQ211637 & DQ211650 & 1956 DRC & Human \\
\hline AP92 & DQ211612 & DQ211625 & DQ211638 & 1975 Greece & Tick \\
\hline TADJ/HU8966 & AY720893 & AY179962 & AY049083 & 1990 Tajikistan & Human \\
\hline ROS/HUVLV-100 & AY995166 & DQ206448 & DQ206447 & 2003 Russian Federation & Human \\
\hline Ibar10200 & AY947891 & AF467768 & U88410 & 1966 Nigeria & Tick \\
\hline Semunya & DQ076412 & DQ094832 & DQ076413 & 1958 Uganda & Human \\
\hline Turkey-Kelkit06 & GQ337055 & GQ337054 & GQ337053 & 2006 Turkey & Human \\
\hline SPU128/81/7 & DQ076414 & DQ157174 & DQ076415 & 1981 South Africa & Tick \\
\hline SPU4/81 & DQ076417 & DQ157175 & DQ076416 & 1981 South Africa & Human \\
\hline Afg09-2990 & HM452307 & HM452306 & HM452305 & 2009 Afghanistan & Human \\
\hline NIVA 118594 & JN572092 & JN572084 & JN572087 & 2011 India & Tick \\
\hline NIV 112143 & JN572091 & JN572085 & JN572089 & 2011 India & Human \\
\hline NIVA 118595 & JN572090 & JN572083 & JN572088 & 2011 India & Tick \\
\hline YL04057 & FJ562095 & FJ562094 & FJ562093 & 2004 China & Tick \\
\hline Sudan AB1-2009 & HQ378183 & HQ378187 & HQ378179 & 2009 Sudan & Human \\
\hline Sudan Al-Fulah 3-2008 & HQ378180 & HQ378184 & GQ862371 & 2008 Sudan & Human \\
\hline $79121 \mathrm{M} 18$ & GU477492 & GU477493 & GU477494 & 1979 China & Rodent \\
\hline
\end{tabular}


Table 3. Summary of southern African CCHFV isolates included in the study

\begin{tabular}{|c|c|c|c|c|c|c|}
\hline \multirow[b]{2}{*}{ Strain } & \multicolumn{3}{|c|}{ Accession numbers } & \multirow{2}{*}{$\begin{array}{l}\text { Year and location } \\
\text { of isolation }\end{array}$} & \multirow{2}{*}{$\begin{array}{l}\text { Source of } \\
\text { human infection }\end{array}$} & \multirow[b]{2}{*}{ Outcome } \\
\hline & $\mathrm{L}$ & $\mathrm{M}$ & $\mathrm{S}$ & & & \\
\hline SPU431/85 & KJ682799 & KJ682812 & KJ682815 & 1985 Northern Cape & Nosocomial & Fatal \\
\hline SPU383/87 & KJ682801 & KJ682806 & KJ682816 & 1987 Free State & Tick & Survived \\
\hline SPU556/87 & KJ682798 & KJ682811 & KJ682817 & 1987 Northern Cape & Tick & Fatal \\
\hline SPU18/88 & KJ682803 & KJ682810 & KJ682818 & 1988 Northern Cape & Tick & Fatal \\
\hline SPU45/88 & KJ682796 & KJ682809 & KJ682819 & 1988 Free State & Tick & Fatal \\
\hline SPU497/88 & KJ682804 & KJ682808 & KJ682820 & 1988 Namibia & Livestock/ tick & Fatal \\
\hline SPU130/89 & KJ682802 & KJ682807 & KJ682821 & 1989 Northern Cape & Tick & Survived \\
\hline SPU48/90 & KJ682797 & KJ682813 & KJ682822 & 1990 North West Province & Unknown & Survived \\
\hline SPU187/90 & KJ682795 & KJ682814 & KJ682823 & 1990 North West Province & Abbatoir & Fatal \\
\hline SPU44/08 & KJ682800 & KJ682805 & KJ682824 & 2008 Free State & Livestock/tick & Survived \\
\hline
\end{tabular}

contigs from both methods were assembled in Geneious (Geneious v. 4.8.5, Biomatters, http:// www.geneious.com/) and compared to known complete CCHFV sequences to identify areas of incomplete coverage or ambiguities which required further investigation. Ambiguities and homopolymers were investigated using Gap5 [23] and the majority could be clarified by evaluation of the quality scores at each relevant position. Where necessary, additional Sanger sequencing was performed and sequences were incorporated into the assembly using Geneious and ChromasPro v. 1.42 (Technelysium Pty Ltd, Australia). Alignments were confirmed by visual inspection with reference sequences and comparison with previously published partial sequences.

Complete genome sequence data for 31 isolates were retrieved from GenBank as summarized in Table 2. The sequences were aligned using Clustal X version 2.0 [24] and analysed using Molecular Evolutionary Genetics Analysis (MEGA) version 5 [25] with the bootstrap maximum-likelihood method with 1000 replicates. Sequence divergence was also determined using MEGA by calculating the average $P$ distances within and between sequence groups as well as pairwise distances for nucleotide and deduced amino acid sequences.

\section{RESULTS}

\section{Sequencing data}

Full coverage of complete $\mathrm{L}, \mathrm{M}$ and $\mathrm{S}$ segments was obtained for 10 southern African CCHFV isolates. The details of the 10 isolates are summarized in Table 3. Ion Torrent PGM sequencing yielded raw data of $>80000$ reads including $>13$ million bases for each of the three isolates. Following stringent filtering and trimming of reads based on length as well as quality scores, $>35000$ reads amounting to $\sim 7$ million bases were included in the final analysis of each of these isolates. The 454 GS Junior sequencing data were more variable and duplicate runs were performed for four of the isolates in order to obtain complete coverage. The raw data for these seven isolates yielded between 2404 and 17315 reads including 1-8 million bases per isolate. Following filtering and trimming, the final alignments were performed on between 1105 and 7042 reads with $~ 500000$ to 3.5 million bases per isolate. The alignments generated by MIRA de novo assembly of both blast-filtered and unfiltered data corresponded, although improved contiguous quality was achieved with some isolates following the application of filter_by_blast. A comprehensive sequence database and optimization of blast parameters were required to ensure effective filtering of reads without loss of coverage, particularly of the highly variable $\mathrm{M}$ segment.

\section{Genetic analysis}

The genetic relationship of isolates as determined by the maximum-likelihood method is shown for the S (Fig. 1a), M (Fig. 1b) and L (Fig. 1c) segments. The phylogenetic groups were designated I-VII as defined previously $[8,26]$. The complete sequences confirm the phylogenetic grouping of southern African isolates SPU497/88, SPU130/89, SPU383/87, SPU18/88 and SPU45/88 and the occurrence of reassortment of the $\mathrm{M}$ segment of these isolates as suggested by partial sequencing [27]. The complete sequences also confirm that the incongruencies in 

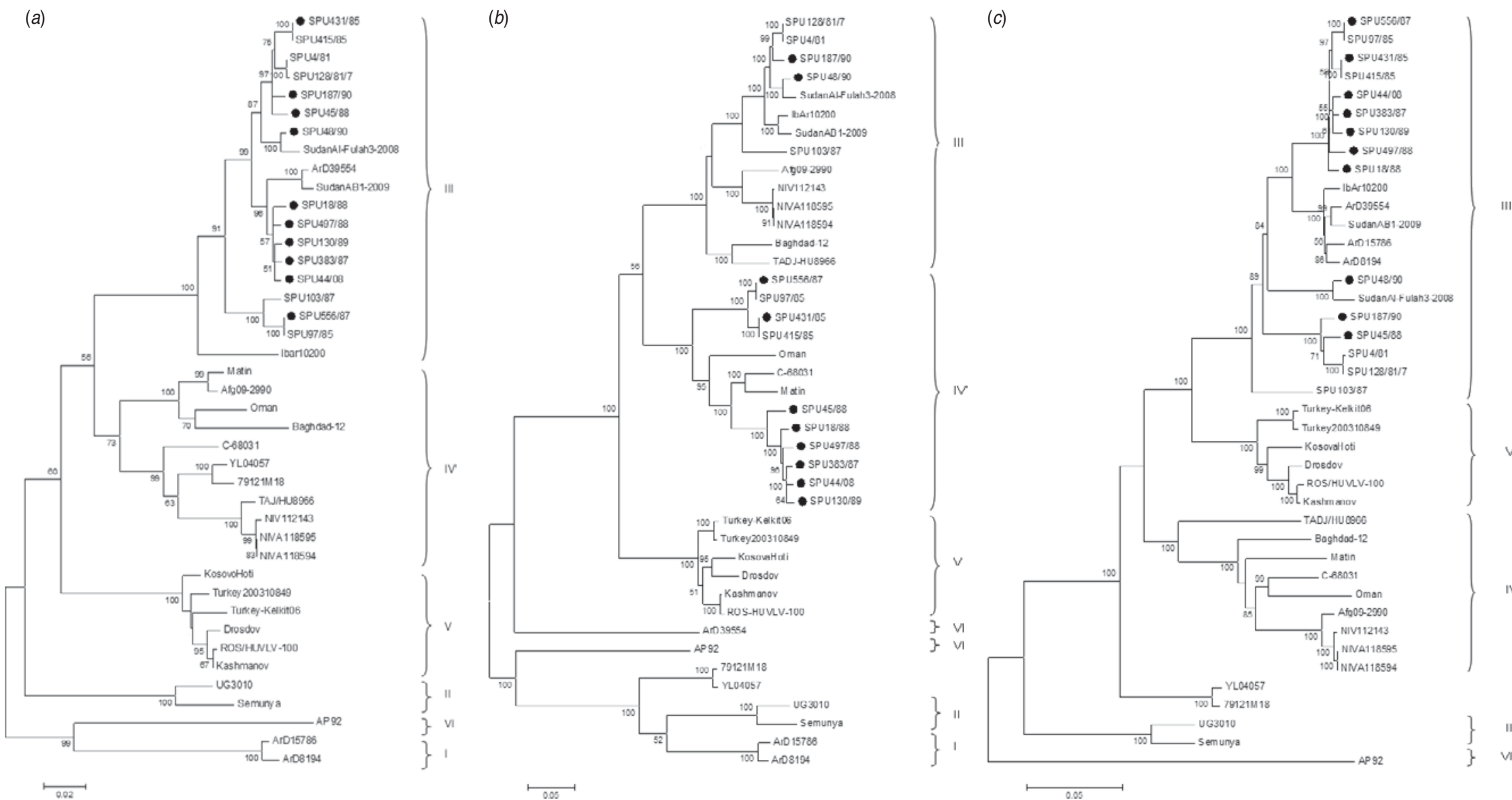

Fig. 1. Phylogenetic analysis of complete coding regions of (a) S segments, (b) M segments, and (c) L segments of CCHFV using a bootstrap maximum-likelihood method with 1000 replicates, with bootstrap values $>50 \%$ indicated at the relevant nodes. Each sequence is designated by the isolate name and isolates sequenced in the current study are indicated by a solid circle $(\mathbf{0})$ 
M segment grouping were due to reassortment rather than recombination events. This pattern of $\mathrm{M}$ segment reassortment, showing clustering of the $\mathrm{S}$ and $\mathrm{L}$ segments with group III strains from South and West Africa while the $\mathrm{M}$ segment clusters with group IV isolates from Asia and the Middle East, was also noted for newly sequenced isolates SPU556/87, SPU44/08 and SPU431/85. Isolates SPU48/90 and SPU187/90 showed no evidence of reassortment, with all three segments falling within the group III South/West Africa lineage. Further evidence of $M$ segment reassortment was seen in southern African isolates SPU97/85 and SPU415/85, as described previously [8]. No evidence of $\mathrm{S}$ segment reassortment was seen in the available complete sequences, but $\mathrm{L}$ segment reassortment was seen in two CCHFV isolates from Senegal, ArD15786 and ArD8194 [8]. The Chinese isolates YL04057 and 79121M18 appear to represent a new group as suggested by Zhou et al. [18], with S segments related to group IV Asia and Middle East, while the M and L segments cluster separately from other known groups. Isolate SPU431/85 was obtained from the same patient but from a sample collected 3 days subsequent to previously published SPU415/85. The sequences correlated well with only a single non-synonymous mutation in the coding region of the $\mathrm{L}$ segment and four non-synonymous mutations in the coding region of the $\mathrm{M}$ segment, as confirmed by Sanger sequencing. RNA from both of these isolates was obtained from cell cultures and these additional passages may have contributed to the accumulated mutations noted.

The geographical distribution of CCHFV groups is illustrated in Figure 2. The S and L segments show a strong correlation in the distinct geographical grouping. The map displaying $M$ segment group distribution clearly illustrates the blending of Asian and African strains, while the remaining groups correlate with $\mathrm{S}$ and $\mathrm{L}$ segment distributions.

The mean $P$ distances within groups and between groups as calculated with MEGA support the phylogenetic groupings. The $\mathrm{S}$ segment nucleotide distances within groups were generally low at between $0 \cdot 1 \%$ and $1.4 \%$ and similar to the amino acid distances within groups which ranged from $0 \cdot 1 \%$ to $1 \cdot 8 \%$. The nucleotide and amino acid distances between $\mathrm{S}$ segment groups were also similar at $2 \cdot 5-6 \cdot 8 \%$ and $2 \cdot 8-8 \cdot 4 \%$, respectively. The $\mathrm{L}$ segment showed a similar degree of diversity within groups at both nucleotide and amino acid levels, but increased diversity between groups. Nucleotide distances within groups ranged from $0 \cdot 4 \%$ to $2 \cdot 5 \%$ and between groups from $3 \cdot 8 \%$ to $11.3 \%$, while amino acid distances within groups were between $0.5 \%$ and $1.8 \%$ and amino acid distances between groups were $2 \cdot 9-10 \cdot 1 \%$. The $\mathrm{M}$ segment showed the greatest genetic diversity, particularly between groups, including numerous non-synonymous mutations. The nucleotide distances within groups ranged from $0 \cdot 4 \%$ to $5 \cdot 5 \%$, while the nucleotide distances between groups were between $10.9 \%$ and $26 \cdot 8 \%$. At the amino acid level, the distances within groups were between $1 \cdot 1 \%$ and $8.3 \%$ and the distances between groups were between $15 \cdot 1 \%$ and $30 \cdot 1 \%$.

Among the southern African isolates, the $\mathrm{S}$ segment was highly conserved with both nucleotide and amino acid mean distances of $<1 \%$ (range $0-1 \cdot 3 \%$ and $0-1 \cdot 7 \%$, respectively). The diversity was also low for the $\mathrm{L}$ group with mean distances of $1.7 \%$ at the nucleotide level (range $0-2 \cdot 8 \%$ ) and $1 \cdot 2 \%$ at the amino acid level (range $0-2 \cdot 1 \%$ ). The diversity of $\mathrm{M}$ segments was higher in group IV isolates than group III, with mean nucleotide distances of $5 \%$ and $2 \cdot 8 \%$, respectively and mean amino acid distances of $7 \cdot 5 \%$ and $4 \cdot 1 \%$, respectively. Overall, the southern African M segments showed distances of $0-12 \cdot 4 \%$ at the nucleotide level and $0-15 \cdot 6 \%$ at the amino acid level.

Although no association between $\mathrm{M}$ segment reassortment and pathogenicity was noted in the southern African isolates included in the current study, the number of both non-fatal infections and nonreassortants included was small. Furthermore, no temporal association was seen with the isolation of reassortants.

\section{DISCUSSION}

The $\mathrm{S}$ segment of CCHFV was previously investigated as a surrogate for complete sequencing due to its length and the fact that it is relatively conserved in comparison to the $\mathrm{M}$ and $\mathrm{L}$ segments thereby simplifying the sequencing process. However, the demonstration of both reassortment $[8,20,27,28]$ and, less commonly, recombination [29] among CCHFV genomes confirmed the necessity of at least partial sequencing of all three segments in order to perform accurate genetic analyses. The present study made use of two NGS platforms, namely the Ion Torrent PGM and the Roche 454 GS Junior sequencing systems, to obtain complete CCHFV sequences for ten southern African isolates. The largest collection of complete CCHFV genome sequences to date made use of $25 \mathrm{~S}, 40 \mathrm{M}$ and $84 \mathrm{~L}$ primers in order to obtain 
(a)

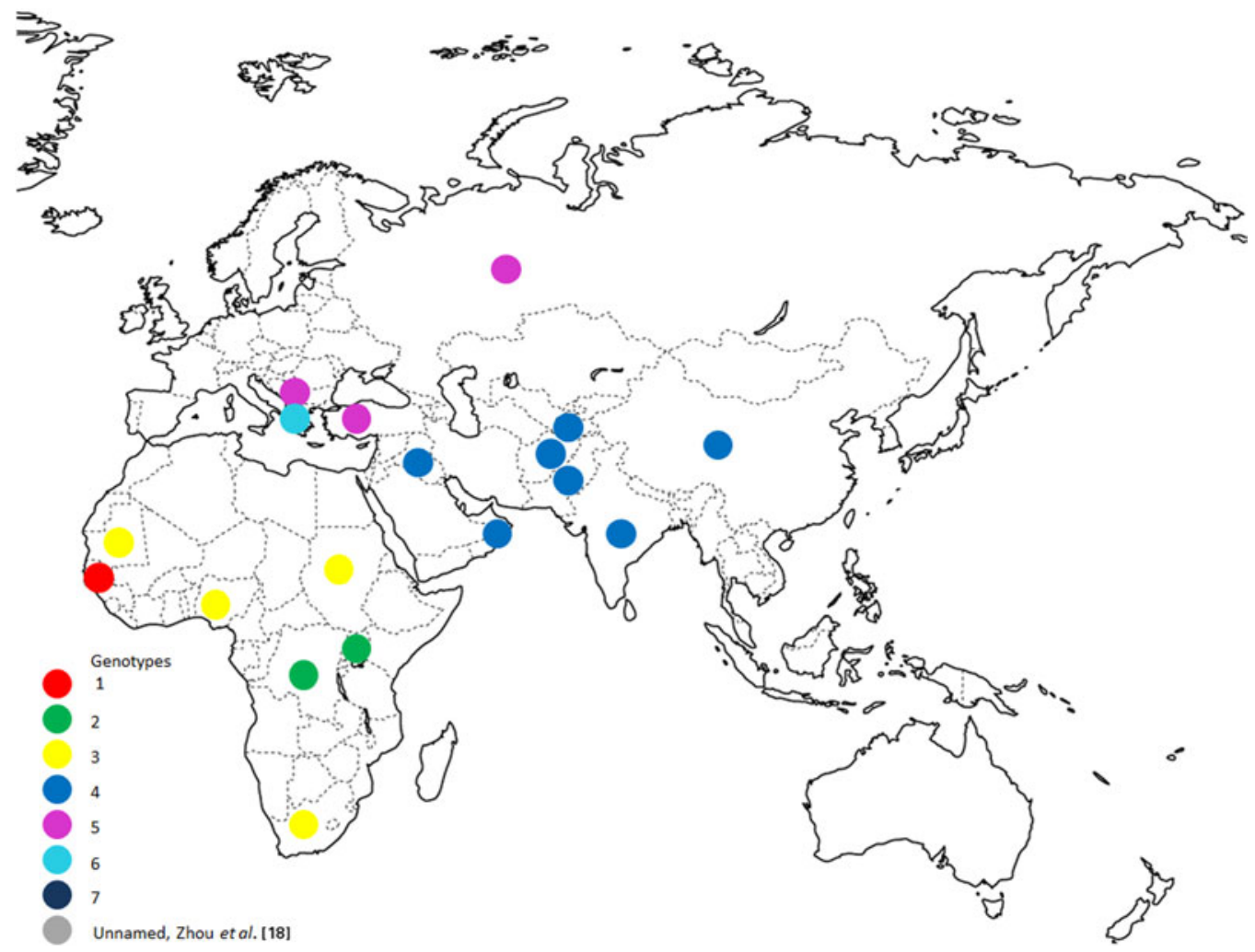

(b)

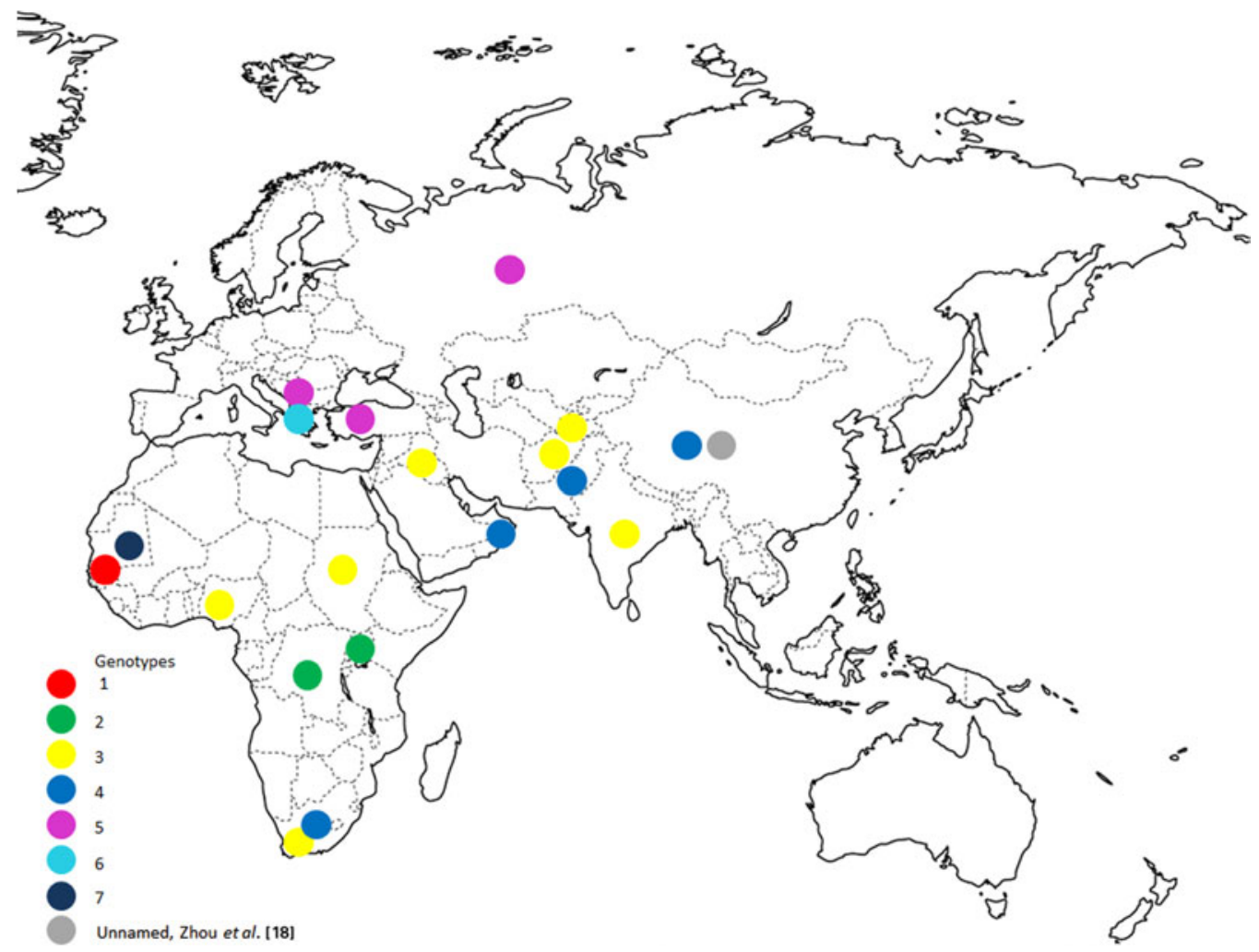

Fig. 2. Geographical distribution of CCHFV groups for the (a) S segment, (b) M segment, and (c) L segment. 


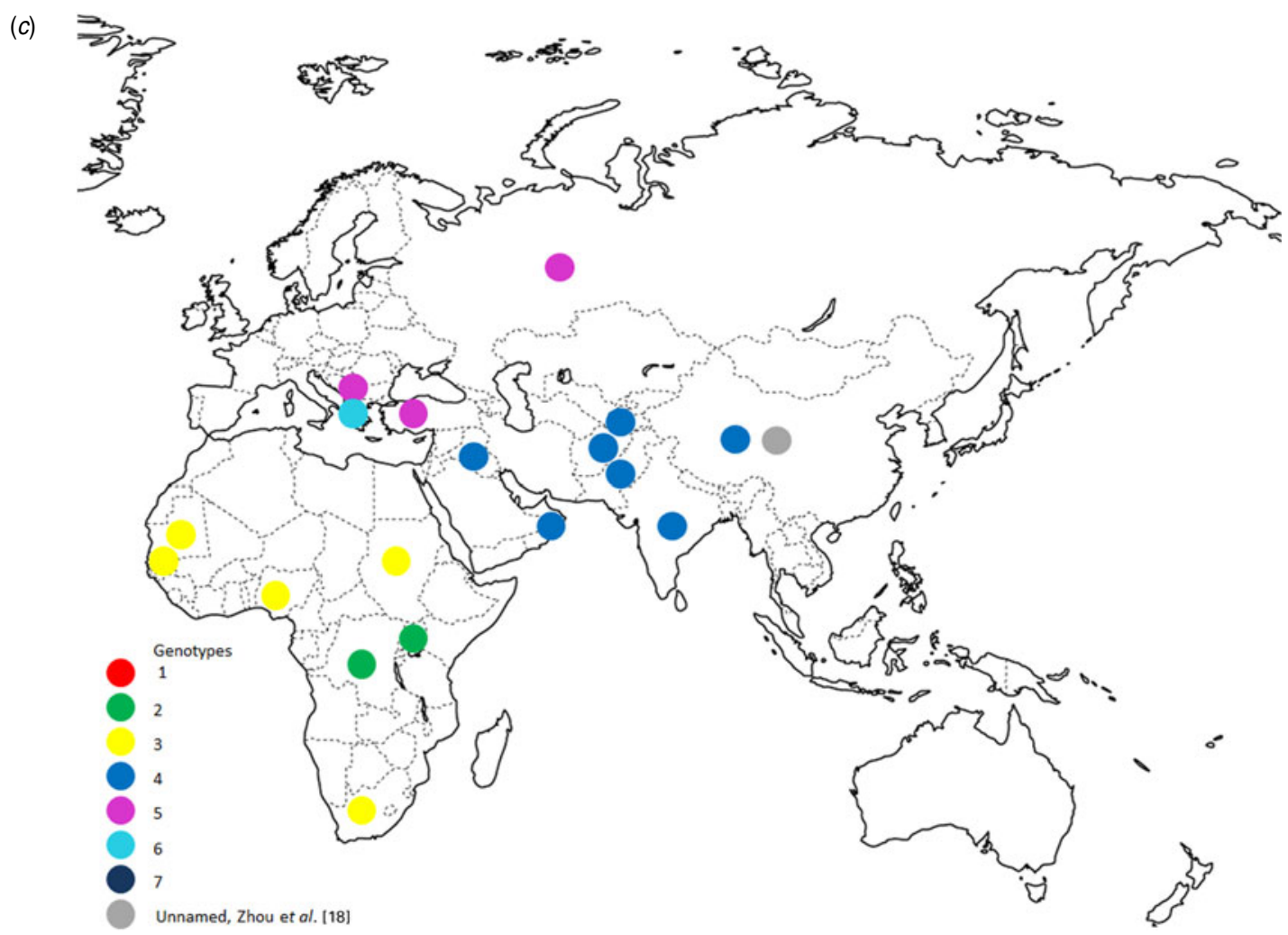

Fig. $2(c)$. For legend see previous page.

complete sequence data for 13 geographically distinct isolates [8]. NGS methods present a relatively simple alternative, requiring only appropriate primers for the generation of amplicons by RT-PCR and potentially a limited number of primers to verify isolated regions or bases. This process could be simplified further by performing NGS of CCHFV directly from clinical samples without prior amplification. This would not only negate the need for specific primers and therefore allow sequencing of diverse CCHFV isolates but would also remove bias introduced by PCR errors [30] as well as multiple passages sometimes required to generate adequate viral titres. This method has been successfully used to sequence RNA viruses such as lyssaviruses directly from tissue samples and cell culture lysates [31]. The introduction of errors during PCR can largely be overcome by using high fidelity enzymes. Although the assembly of raw data generated by NGS platforms can be complex, the workflow described in the present study made use almost exclusively of open source software and could be applied to a range of datasets from various sources. Confirmation of the validity of the assemblies obtained was made possible by comparison with a number of partial sequences which were available from a previous study [27], confirming the accuracy of the methods used.

Genetic evolution of arboviruses, including $\mathrm{CCHFV}$, is a complex process influenced by multiple factors. As with other RNA viruses, the RNAdependent RNA polymerases lack proofreading activity and show error frequencies of $\sim 10^{-4}$ [32]. This is offset by the effect of alternating infections of arthropods and vertebrates which constrains virus adaptation of arboviruses in comparison to other RNA viruses [33]. Despite this, CCHFV shows a high level of genetic variability. Inclusion of diverse isolates in the current study, particularly from China and central and West Africa, led to nucleotide variation of up to $7 \%, 27 \%$ and $11 \%$ for the $\mathrm{S}, \mathrm{M}$ and $\mathrm{L}$ segments, respectively, and amino acid variation of up to $8 \%, 30 \%$ and $10 \%$. This is similar to previous studies with greater variability of the $M$ and $L$ segments compared to the $\mathrm{S}$ segment, which is contrary to expectation as the viral RNA polymerase is usually highly conserved $[8,34]$.

Tree topologies for southern African isolates based on partial S, M and L sequence data correlated with topologies constructed using complete genome data (data available from corresponding author on 
request), provide further evidence that incongruencies in grouping are likely reassortant events and not recombination [27]. Genetic relationships indicate movement of CCHFV isolates within and between continents. Isolates from the same group can be found in geographically distinct locations and isolates from different groups can be found to co-circulate in similar regions. Genetic diversity within regions supports movement of the virus by bird migration and livestock trade. Reassortment events have occurred between West African and southern African isolates and between southern African and Asian isolates. Interestingly, the reassortment events between West Africa and southern Africa involved the L segment, whereas reassortment events between southern Africa and Asia involved the M segment. This may be related to the tick species found in these geographical areas. The mechanism of reassortment is unclear; however, it is assumed to occur in the tick host where dual persistent infections are more likely. Few complete sequences are available from West Africa but as three of the four available isolates show either $\mathrm{L}$ or $\mathrm{M}$ segment reassortment, it appears that reassortant viruses may occur frequently in this area. The two novel Chinese isolates described by Zhou et al. cluster independently in an as yet unnamed group, suggesting the occurrence of further genetic groups [18]. Additional complete genome sequence data from geographically distinct isolates are required to corroborate and expand these findings.

Reassortment is widely described for members of the Bunyaviridae family [35]. Both homologous and heterologous reassortment may occur and may result in altered viral phenotypes. Ngari virus is a reassortant of Bunyamwera and Batai viruses and is associated with haemorrhagic fever, in contrast to the mild disease associated with the parent viruses [36]. Both increased neuroinvasiveness and enhanced transmission by insect vectors have been specifically linked to the $\mathrm{M}$ segment of La Crosse virus, another member of the Bunyaviridae family [37, 38]. Burt et al. suggested a possible association between $\mathrm{M}$ segment reassortment of CCHFV and pathogenicity although this finding was not statistically significant [27]. The current study could not confirm this association but given the small number of isolates included, further investigation is warranted. As 10 of the 15 complete sequences of CCHFV from southern Africa show $\mathrm{M}$ segment reassortment, it would be of interest to determine whether these $M$ segments provide a competitive advantage such as the increased transmissibility demonstrated in La Crosse virus reassortants. The postulated co-evolution of $\mathrm{S}$ and $\mathrm{L}$ segments, possibly related to functional interdependency [7], may account for the relative scarcity of reassortment of these segments.

It is likely that both genetic drift and shift of CCHFV genomes occur chiefly during infection of insect vectors rather than in mammalian hosts due to the longer period of infection and increased likelihood of super-infection with more than one strain $[11,39]$. In contrast to the short period of viraemia seen in humans [40], ticks remain infected over much longer periods with transstadial and, more rarely, transovarial transmission playing an important role in virus perpetuation and therefore also virus evolution [39, 41, 42].

In conclusion, NGS methods provide a simple and effective alternative to Sanger sequencing for the determination of complete CCHFV genomes. The application of these methods to clinical samples without prior PCR amplification would provide further advantages and should be validated on CCHFV isolates. Our genetic analysis of complete genome sequences confirms the high frequency of $\mathrm{M}$ segment reassortment in southern African CCHFV isolates. Further studies are indicated to elucidate the possible consequences of reassortment particularly relating to viral pathogenicity and transmissibility.

\section{DECLARATION OF INTEREST}

None.

\section{ACKNOWLEDGEMENTS}

We thank Anelda van der Walt, Central Analytical Facility, Stellenbosch University for advice regarding NGS analysis. This project was funded by the National Health Laboratory Service Research Trust, the Polio Research Foundation, South Africa, and University of the Free State Cluster funding.

\section{REFERENCES}

1. Elliott RM, et al. Family Bunyaviridae. In Regenmortel MHV, Fauquet CM, Bishop DHL, Carstens EB, Estes MK, Lemon SM, Maniloff J, Mayo MA, McGeoch DJ, Pringle CR and Wickner RB, eds. Seventh Report of the International Committee on Taxonomy of Viruses. San Diego: Academic Press, 2000, pp. 599-621.

2. Schmaljohn CS, Hooper JW. Bunyaviridae: the viruses and their replication. In: Knipe DM and Howley PM, 
eds. Fields Virology, Volume 1, 4th edn. Philadelphia: Lippincott, Williams \& Wilkins, 2001, pp. 1581-1602.

3. Whitehouse CA. Crimean-Congo hemorrhagic fever. Antiviral Research 2004; 64: 145-160.

4. Bente DA, et al. Crimean-Congo hemorrhagic fever: History, epidemiology, pathogenesis, clinical syndrome and genetic diversity. Antiviral Research 2013; 100: 159-189.

5. Msimang V, et al. Update: Crimean-Congo haemorrhagic fever in South Africa. Communicable Diseases Surveillance Bulletin 2013; 11: 62-65.

6. Aradaib IE, et al. Multiple Crimean-Congo hemorrhagic fever virus strains are associated with disease outbreaks in Sudan, 2008-2009. PLoS Neglected Tropical Diseases 2011; 5: e1159.

7. Chamberlain J, et al. Co-evolutionary patterns of variation in small and large RNA segments of CrimeanCongo hemorrhagic fever virus. Journal of General Virology 2005; 86: 3337-3341.

8. Deyde VM, et al. Crimean-Congo hemorrhagic fever virus genomics and global diversity. Journal of Virology 2006; 80: 8834-8842.

9. Duh D, et al. The complete genome sequence of a Crimean-Congo hemorrhagic fever virus isolated from an endemic region in Kosovo. Virology Journal 2008; 5: 7 .

10. Hewson R, et al. Evidence of segment reassortment in Crimean-Congo haemorrhagic fever virus. Journal of General Virology 2004; 85: 3059-3070.

11. Kinsella E, et al. Sequence determination of the Crimean-Congo hemorrhagic fever virus L segment. Virology 2004; 321: 23-28.

12. Meissner JD, et al. The complete genome sequence of strain ROS/HUVLV-100, a representative Russian Crimean Congo hemorrhagic fever virus strain. Virus Genes 2006; 33: 87-93.

13. Olschläger S, et al. Complete sequence and phylogenetic characterisation of Crimean-Congo hemorrhagic fever virus from Afghanistan. Journal of Clinical Virology 2011; 50: 90-92.

14. Ozdarendeli A, et al. The complete genome analysis of Crimean-Congo hemorrhagic fever virus isolated in Turkey. Virus Research 2010; 147: 288-293.

15. Papa A, et al. Genetic characterization of the M RNA segment of Crimean Congo hemorrhagic fever virus strains, China. Emerging Infectious Diseases 2002; 8:50-53.

16. Seregin SV, et al. Genetic characterization of the $\mathrm{M}$ RNA segment of Crimean-Congo hemorrhagic fever virus strains isolated in Russia and Tajikistan. Virus Genes 2004; 28: 187-193.

17. Yadav PD, et al. Genetic characterization and molecular clock analyses of the Crimean-Congo hemorrhagic fever virus from human and ticks in India, 2010-2011. Infection, Genetics and Evolution 2013; 14: 223-231.

18. Zhou A, et al. Complete genome sequences of two Crimean-Congo hemorrhagic fever viruses isolated in China. Genome Announcements 2013; 1: e00571-13.

19. Capobianchi MR, Giombini E, Rozera G. Next-generation sequencing technology in clinical virology. Clinical Microbiology and Infection 2013; 19: 15-22.

20. Burt FJ, Swanepoel R. Molecular epidemiology of African and Asian Crimean-Congo haemorrhagic fever isolates. Epidemiology and Infection 2005; 133: 659-666.

21. Schmieder R, Edwards R. Quality control and preprocessing of metagenomic datasets. Bioinformatics 2011; 27: 863-864.

22. Chevreux B, Wetter T, Suhai S. Genome sequence assembly using trace signals and additional sequence information. In Computer Science and Biology: Proceedings of the German Conference on Bioinformatics (GCB), 1999, pp. 45-56.

23. Bonfield JK, Smith KF, Staden R. A new DNA sequence assembly program. Nucleic Acids Research 1995; 23: 4992-4999.

24. Larkin MA, et al. Clustal W and Clustal X version 2.0. Bioinformatics 2007; 23: 2947-2948.

25. Tamura K, et al. MEGA5: Molecular Evolutionary Genetics Analysis using maximum likelihood, evolutionary distance, and maximum parsimony methods. Molecular Biology and Evolution 2011; 28: 2731-2739.

26. Carroll SA, et al. Ancient common ancestry of Crimean-Congo heamorrhagic fever virus. Molecular Phylogenetics and Evolution 2010; 55: 1103-1110.

27. Burt FJ, et al. Genetic relationship in southern African Crimean-Congo haemorrhagic fever isolates: evidence for occurrence of reassortment. Epidemiology and Infection 2009; 137: 1302-1308.

28. Grard G, et al. Re-emergence of Crimean-Congo hemorrhagic fever virus in Central Africa. PLoS Neglected Tropical Diseases 2011; 5: e1350.

29. Lukashev AN. Evidence for recombination in Crimean-Congo hemorrhagic fever virus. Journal of General Virology 2005; 86: 2333-2338.

30. Bracho MA, Moya A, Barrio E. Contribution of Taq polymerase-induced errors to the estimation of RNA virus diversity. Journal of General Virology 1998; 79: 2921-2928.

31. Marston DA, et al. Next generation sequencing of viral RNA genomes. BMC Genomics 2013; 14: 444.

32. Steinhauer DA, Domingo E, Holland JJ. Lack of evidence for proofreading mechanisms associated with an RNA virus polymerase. Gene 1992; 122: 281-288.

33. Coffey LL, et al. Arbovirus evolution in vivo is constrained by host adaptation. Proceedings of the National Academy of Sciences USA 2008; 105: 6970 6975.

34. Anagnostou V, Papa A. Evolution of Crimean-Congo hemorrhagic fever virus. Infection, Genetics and Evolution 2009; 9: 948-954.

35. Briese T, Calisher $\mathbf{C H}$, Higgs $\mathbf{S}$. Viruses of the family Bunyaviridae: are all available isolates reassortants? Virology 2013; 446: 207-216.

36. Bowen MD, et al. A reassortant bunyavirus isolated from acute hemorrhagic fever cases in Kenya and Somalia. Virology 2001; 291: 185-190.

37. Beaty BJ, et al. Molecular basis of bunyavirus transmission by mosquitoes: role of the middle-sized RNA segment. Science 1981; 211: 1433-1435. 
38. Gonzalez-Scarano F, et al. Genetics infectivity and virulence of California serogroup viruses. Virus Research 1992; 24: 123-135.

39. Hoogstraal H. The epidemiology of tick-borne CrimeanCongo haemorrhagic fever in Asia, Europe, and Africa. Journal of Medical Entomology 1979; 15: 307-417.

40. Shepherd AJ, et al. Comparison of methods for isolation and titration of Crimean-Congo hemorrhagic fever virus. Journal of Clinical Microbiology 1986; 24: 654-656.
41. Gonzalez JP, et al. Sexual and transovarian transmission of Crimean-Congo haemorrhagic fever virus in Hyalomma truncatum ticks. Research in Virology 1992; 143: 23-28.

42. Gordon SW, Linthicum KJ, Moulton JR. Transmission of Crimean-Congo hemorrhagic fever virus in two species of Hyalomma ticks from infected adults to cofeeding immature forms. American Journal of Tropical Medicine and Hygiene 1993; 48: 576-580. 